COMPUTATIONAL METHODS IN APPLIED MATHEMATICS, Vol.1(2001), No. 1, pp. 99-112

(C) 2001 Editorial Board of the Journal "Computational Methods in Applied Mathematics" Joint Co. Ltd.

\title{
MULTIGRID FOR THE WILSON MORTAR ELEMENT METHOD
}

\author{
ZHONG-CI SHI AND XUEJUN XU \\ Institute of Computational Mathematics, Academy of Mathematics and System Sciences \\ Chinese Academy of Sciences, P.O.Box 2719, Beijing 100080, China \\ E-mail: shi@lsec.cc.ac.cn, xxj@lsec.cc.ac.cn
}

\begin{abstract}
In this paper, a mortar finite element method is proposed for the Wilson nonconforming element. Multigrid method is used to solve the resulting discrete system.
\end{abstract}

2000 Mathematics Subject Classification: 65F10, 65N30.

Keywords: mortar finite element method, Wilson nonconforming element, multigrid method.

\section{Introduction}

In recent years, the mortar finite element method as a special domain decomposition methodology appears very attractive because it can handle the situations where meshes on different subdomains need not align across interfaces, and the matching of discretizations on adjacent subdomains is only enforced weakly. In [6], Bernardi, Maday and Patera first introduced basic concepts of general mortar element method, including the coupling of spectral elements with finite elements. Then it was extensively used and analyzed by many authors. In [4], Belgacem studied the mortar elment method under a primal hybrid finite element formulation. Meanwhile, some extensions and convergence results in three dimensions were considered in $[5,7]$. Recently, many works have been done in constructing efficient iteration solvers for the discrete system resulting from the mortar element method. The first approches were based on iterative substructuring methods, see $[1-3,12]$. Multigrids for the mortar element method have also been considered. Gopalakrishnan and Pasciak [13] presented a variable $V$-cycle preconditioner, while Braess, Dahmen and Wieners [8] and Wohlmuth [17] established a $W$-cycle multigrid based on a hybrid formulation which gives rise to a saddle point problem.

Until now, the mortar finite element method is mainly concerned with discretization of conforming elements. There only few papers concern nonconforming elements, e.g., Marcinkowski [14] presented the $P_{1}$ nonconforming mortar element. In this paper, we will study the mortar finite element method for the Wilson nonconforming element. This element has been widely used in computational mechanics and structural engineering because it holds a better convergence behavior than the bilinear element (cf. [16,18] for details). We first design a mortar condition for the Wilson element. Based on this condition, we obtain an optimal energy norm error estimation. Moreover, we will present a multigrid algorithm for solving the resulting algebraic system. It is proved that a $W$-cycle multigrid method is optimal, i.e., 
the convergence rate is independent of the mesh size and mesh level. Meanwhile, a variable $V$-cycle multigrid preconditioner is constructed, which results in a preconditioned system with a uniformly bounded condition number.

This paper is organized as follows. In Sections 2 and 3, a model problem and the Wilson mortar finite element method are described. In Section 4, we present our multigrid algorithm. Its convergence analysis is given in Section 5 .

\section{A model problem}

For simplicity, we consider a model second order problem

$$
\left\{\begin{aligned}
-\triangle u=f & \text { in } \quad \Omega \\
u=0 & \text { on } \quad \partial \Omega
\end{aligned}\right.
$$

where $\Omega \subset R^{2}$ is a bounded polygonal domain, $f \in L^{2}(\Omega)$. There is no difficulty in extending the results in this paper to more general second order elliptic problems.

The variational form of $(1)$ is to find $u \in H_{0}^{1}(\Omega)$ such that

$$
a(u, v)=(f, v), \quad \forall v \in H_{0}^{1}(\Omega)
$$

where the bilinear form

$$
a(u, v)=\int_{\Omega} \nabla u \cdot \nabla v d x d y, \quad \forall u, v \in H^{1}(\Omega) .
$$

Assume that the problem (2) has the following regularity.

(H1). For any $f \in L^{2}(\Omega)$, it holds that

$$
\|u\|_{2} \leqslant C\|f\|_{0} .
$$

\section{The Wilson mortar finite element method}

First, we divide $\Omega$ into nonoverlapping rectangular subdomains such that

$$
\bar{\Omega}=\bigcup_{i=1}^{N} \bar{\Omega}_{i} \quad \text { and } \quad \Omega_{i} \cap \Omega_{j}=\varnothing, \quad i \neq j .
$$

They are so arranged that the intersection of $\Omega_{i} \cap \Omega_{j}$, for $i \neq j$ is either an empty set, an edge or a vertex, i.e., the partition is geometrically conforming. The interface

$$
\Gamma=\bigcup_{i=1}^{N} \partial \Omega_{i} \backslash \partial \Omega
$$

is broken into a set of disjoint open straight segments $\gamma_{m}(1 \leqslant m \leqslant M)$ (that are the edges of subdomains) called mortars, i.e.,

$$
\Gamma=\bigcup_{k=1}^{M} \bar{\gamma}_{m}, \quad \gamma_{m} \cap \gamma_{n}=\varnothing, \quad \text { if } m \neq n .
$$


We denote the common open edge for $\Omega_{i}$ and $\Omega_{j}$ by $\gamma_{m}$. By $\gamma_{m(i)}$ we denote the edge of $\Omega_{i}$ which is called a mortar and by $\delta_{m(j)}$ the edge of $\Omega_{j}$ which geometrically occupies the same place and is called nonmortar.

Let $\Gamma_{1}^{i}$ be the coarsest triangulation of $\Omega_{i}$ with the mesh size $h_{1}$. Generally the triangulation does not align at the subdomain interface. Denote the global mesh $\cup_{i} \Gamma_{1}^{i}$ by $\Gamma_{1}$. We refine the triangulation $\Gamma_{1}$ to produce $\Gamma_{2}$ by joining the midpoints of opposite edges of rectangles in $\Gamma_{1}$. Obviously, the mesh size $h_{2}$ in $\Gamma_{2}$ is $h_{2}=h_{1} / 2$. Repeating this process, we get the $l$-time refined triangulation $\Gamma_{l}$ with the mesh size $h_{l}=h_{1} 2^{-l}(l=1, \ldots, L)$.

Define

$$
X=\left\{v|v|_{\Omega_{i}} \in H^{1}\left(\Omega_{i}\right), \quad \forall i=1, \ldots, N, v=0 \text { on } \partial \Omega\right\} .
$$

On each level $l$, we define the Wilson element space locally and introduce the space

$$
V_{l, i}=\left\{v_{l, i}\left|v_{l, i}\right|_{K} \in V(K) \text { and equal to zero at nodes on } \partial \Omega\right\},
$$

where $V(K)$ is the Wilson element space over the rectangle $K \in \Gamma_{l}^{i}$. Usually, the space $V(K)$ is defined as follows:

First, on the reference rectangle $\hat{K}=[-1,1] \times[-1,1]$, the shape function of Wilson element is a quadratic polynomial $\hat{p}$, defined by the values of $\hat{p}$ at four vertices of $\hat{K}$ and the mean values of $\frac{\partial^{2} \hat{p}}{\partial \xi^{2}}$ and $\frac{\partial^{2} \hat{p}}{\partial \eta^{2}}$ on $\hat{K}$. We have

$$
\begin{aligned}
\hat{p}= & \frac{(1+\xi)(1+\eta)}{4} p_{1}+\frac{(1-\xi)(1+\eta)}{4} p_{2}+\frac{(1-\xi)(1-\eta)}{4} p_{3}+\frac{(1+\xi)(1-\eta)}{4} p_{4} \\
& +\frac{1}{2}\left(\xi^{2}-1\right) \frac{\partial^{2} \hat{p}}{\partial \xi^{2}}+\frac{1}{2}\left(\eta^{2}-1\right) \frac{\partial^{2} \hat{p}}{\partial \eta^{2}}
\end{aligned}
$$

where $p_{i}$ is the value of $\hat{p}$ at the vertex $\hat{A}_{i}$.

Then, for each rectangle $K \in \Gamma_{l}^{i}$, using an affine transformation, we can define the Wilson element space $V(K)$. We denote the Wilson finite element space $V_{l, i}\left(\Omega_{i}\right)$ on $\Gamma_{l}^{i}$ by $V_{l, i}$. It is known that this element is not continuous on each interelement boundary, so it is nonconforming for a second order problem.

For any $v \in V_{l, i}$, we can decompose it as $v=\bar{v}+v^{\prime}$, where $\bar{v}$ is the bilinear term of $v$ which is continuous across element boundaries, and so it is a conforming part. The second part $v^{\prime}$ is nonconforming.

Let

$$
\tilde{V}_{l}=\prod_{i=1}^{N} V_{l, i}=\left\{v_{l}\left|v_{l}\right|_{\Omega_{i}}=v_{l, i} \in V_{l, i}\right\} .
$$

It is obvious that

$$
\tilde{V}_{1} \not \subset \nsubseteq \tilde{V}_{L}
$$

The bilinear finite element space over the triangulation $\Gamma_{l}^{i}$ is denoted by $W_{l, i}$, whose functions are equal to zero on $\partial \Omega$. Let

$$
\tilde{W}_{l}=\prod_{i=1}^{N} W_{l, i}=\left\{v_{l}\left|v_{l}\right|_{\Omega_{i}}=v_{l, i} \in W_{l, i}\right\}
$$

for all $l=1, \ldots, L$.

It is easy to see that

$$
\tilde{W}_{1} \subseteq \cdots \subseteq \tilde{W}_{L}
$$


and

$$
\tilde{W}_{l} \subset \tilde{V}_{l} .
$$

For any interface $\gamma_{m}=\gamma_{m(i)}=\delta_{m(j)}, 1 \leqslant m \leqslant M$, there are two different and independent 1D triangulations with the mesh size $h_{l, i}$ and $h_{l, j}$. Let $M_{l}\left(\gamma_{m(i)}\right)$ and $M_{l}\left(\delta_{m(j)}\right)$ be piecewise continuous linear function spaces corresponding to the triangulations $\Gamma_{l}^{i}$ and $\Gamma_{l}^{j}$ restricted to $\gamma_{m}$, respectively. In addition, we define an auxiliary test space $S_{l}\left(\delta_{m(j)}\right)$ as a subspace of the non-mortar space $M_{l}\left(\delta_{m(j)}\right)$ such that its functions are constant on elements that intersect the ends of $\delta_{m(j)}$. Then we have the following Wilson mortar finite element space:

$$
V_{l}=\left\{v_{l} \in \tilde{V}_{l} \mid \forall \delta_{m(j)} \subset \Gamma, \int_{\delta_{m(j)}}\left(\bar{v}_{l, j}-\bar{v}_{l, i}\right) \varphi d s=0 \quad \forall \varphi \in S_{l}\left(\delta_{m(j)}\right)\right\} .
$$

For the following analysis, we need an auxiliary bilinear mortar element space:

$$
W_{l}=\left\{v_{l} \in \tilde{W}_{l} \mid \forall \delta_{m(j)} \subset \Gamma, \int_{\delta_{m(j)}}\left(v_{l, j}-v_{l, i}\right) \varphi d s=0 \quad \forall \varphi \in S_{l}\left(\delta_{m(j)}\right)\right\} .
$$

It is not difficult to check that

$$
W_{l} \subset V_{l} \text {. }
$$

Define

$$
\begin{gathered}
\|v\|_{l, i}^{2} \hat{=} a_{l, i}(v, v) \hat{=} \sum_{K \in \Gamma_{l}^{i}} \int_{K} \nabla v \cdot \nabla v d x d y, \quad \forall v \in V_{l, i}, \\
a_{l}(u, v) \hat{=} \sum_{i=1}^{N} a_{l, i}(v \cdot v), \quad\|v\|_{l}^{2} \hat{=} \sum_{i=1}^{N}\|v\|_{l, i}^{2} .
\end{gathered}
$$

Then the Wilson mortar element approximation of the problem (2) is to find $u_{l} \in V_{l}$ such that

$$
a_{l}\left(u_{l}, v_{l}\right)=f\left(v_{l}\right), \quad \forall v_{l} \in V_{l}
$$

where

$$
f\left(v_{l}\right)=\sum_{i=1}^{N} \int_{\Omega_{i}} f v_{l} d s
$$

Lemma 3.1. $\|\cdot\|_{l}$ defined by (13) is a norm over the space $V_{l}$ and there exists a positive constant $C$ independent of $h_{l}$ such that

$$
\left|a_{l}(u, v)\right| \leqslant C\|u\|_{l}\|v\|_{l} \quad \forall u, v \in V_{l} .
$$

Proof. It is easy to prove (15) by the Cauchy-Schwarz inequality. In fact, let $v_{l}$ be a function in the space $V_{l}$ satisfying

$$
\left\|v_{l}\right\|_{l}^{2}=\sum_{i=1}^{N} \sum_{K \in \Gamma_{l}^{i}}\left|v_{l, i}\right|_{1, K}^{2}=0
$$

Then each $v_{l, i}$ is a constant on $K \in \Gamma_{l}^{i}$. Therefore, the function $v_{l, i}$ is identical to zero on each $\bar{\Omega}_{i}$ where $\bar{\Omega}_{i} \cap \partial \Omega \neq 0$. Moreover, by the mortar matching condition for $V_{l}$, we know that $v_{l}$ is zero everywhere on $\bar{\Omega}$. 
We now give an abstract theorem.

Lemma 3.2. Let $u$ and $u_{l}$ be the solutions of (2) and (14), respectively, then

$$
\left\|u-u_{l}\right\|_{l} \leqslant C\left(\inf _{v_{l} \in V_{l}}\|u-v\|_{l}+\sup _{w_{l} \in V_{l}} \frac{\left|D_{l}\left(u, w_{l}\right)+M_{l}\left(u, w_{l}\right)\right|}{\left\|w_{l}\right\|_{l}}\right)
$$

where

$$
\begin{aligned}
& D_{l}\left(u, w_{l}\right)=\sum_{i=1}^{N} \sum_{K \in \Gamma_{l}^{i}} \int_{\partial K} \frac{\partial u}{\partial n}\left(w_{l}-\bar{w}_{l}\right) d s, \\
& M_{l}\left(u, w_{l}\right)=\sum_{\gamma_{m} \in \Gamma_{\gamma_{m}}} \int_{\frac{\partial u}{\partial n}}\left[\bar{w}_{l}\right] d s,
\end{aligned}
$$

where [.] is a jump function across $\gamma_{m}, n$ is the unit outward normal vector along $\partial K$.

Proof. By the Strang Lemma, we can obtain

$$
\left\|u-u_{l}\right\|_{l} \leqslant C\left(\inf _{v_{l} \in V_{l}}\|u-v\|_{l}+\sup _{w_{l} \in V_{l}} \frac{\left|a_{l}\left(u, w_{l}\right)-f\left(w_{l}\right)\right|}{\left\|w_{l}\right\|}\right) .
$$

It is not difficult to check that

$$
\begin{aligned}
& a_{l}\left(u, w_{l}\right)-\left(f, w_{l}\right) \\
& =\sum_{i=1}^{N} \sum_{K \in \Gamma_{l}^{i} \partial K} \int_{\frac{\partial u}{\partial n}} w_{l} d s \\
& =\sum_{i=1}^{N} \sum_{K \in \Gamma_{l}^{i} \partial K} \int \frac{\partial u}{\partial n}\left(w_{l}-\bar{w}_{l}\right) d s+\sum_{i=1}^{N} \sum_{K \in \Gamma_{l}^{i} \partial K} \int \frac{\partial u}{\partial n} \bar{w}_{l} d s \\
& =\sum_{i=1}^{N} \sum_{K \in \Gamma_{l}^{i} \partial K} \int_{\frac{\partial u}{\partial n}}\left(w_{l}-\bar{w}_{l}\right) d s+\sum_{1 \leqslant m \leqslant M_{\gamma_{m}}} \int_{\frac{\partial u}{\partial n}}\left[\bar{w}_{l}\right] d s,
\end{aligned}
$$

which completes the proof.

Define the space $W_{l}\left(\delta_{m(j)}\right)$ by

$$
\begin{gathered}
W_{l}\left(\delta_{m(j)}\right)=\left\{v \mid v \text { is a linear continuous function on } \delta_{m(j)} \text {, and } v\right. \text { is } \\
\text { vanishing at endpoints of } \left.\delta_{m(j)}\right\} .
\end{gathered}
$$

Accordingly, we define the projection operator $\pi_{l, m}: L^{2}(\Omega) \rightarrow W_{l}\left(\delta_{m(j)}\right)$ as follows [13]:

$$
\int_{\delta_{m(j)}}\left(\pi_{l, m} v\right) \chi d s=\int_{\delta_{m(j)}} u \chi d s, \quad \forall \chi \in S_{l}\left(\delta_{m(j)}\right) .
$$

This projection is known to be stable in $L^{2}\left(\gamma_{m}\right)$ and $H_{00}^{1 / 2}\left(\gamma_{m}\right)[6,8]$, i.e.,

$$
\begin{aligned}
\left\|\pi_{l, m} v\right\|_{0, \gamma_{m}} & \leqslant C\|v\|_{0, \gamma_{m}}, \\
\left\|\pi_{l, m} v\right\|_{H_{00}^{1 / 2}\left(\gamma_{m}\right)} & \leqslant C\|v\|_{H_{00}^{1 / 2}\left(\gamma_{m}\right)} .
\end{aligned}
$$

To obtain the error estimate of the solution $u_{l}$, we give some auxiliary lemmas.

Based on a similar argument as in [6], we can prove that 
Lemma 3.3 (Approximation error). Assume that $v \in H_{0}^{1}(\Omega)$ is such that for any $i, 1 \leqslant i \leqslant N, v_{i}=\left.v\right|_{\Omega_{i}}$ belongs to $H^{2}\left(\Omega_{i}\right)$, then

$$
\inf _{v_{l} \in V_{l}}\left\|v-v_{l}\right\|_{l} \leqslant C\left(\sum_{i=1}^{N} h_{l, i}^{2}\left\|v_{i}\right\|_{2, \Omega_{i}}^{2}\right)^{1 / 2}
$$

where $h_{l, i}=\max _{K \in \Gamma_{l}^{i}} h_{K}, h_{K}$ is the diamater of the rectangle $K \in \Gamma_{l}^{i}$.

Moreover, we have

Lemma 3.4 (Consistency error). Assume that $u$ is such that for any $i, 1 \leqslant i \leqslant N$, $u_{i}=\left.u\right|_{\Omega_{i}}$ belongs to $H^{2}\left(\Omega_{i}\right)$, then

$$
\begin{aligned}
& \text { (1). }\left|D_{l}\left(u, w_{l}\right)\right| \leqslant C\left(\sum_{i=1}^{N} h_{l, i}^{2}\left\|u_{i}\right\|_{2, \Omega_{i}}^{2}\right)^{1 / 2}\left\|w_{l}\right\|_{l}, \\
& \text { (2). }\left|M_{l}\left(u, w_{l}\right)\right| \leqslant C\left(\sum_{i=1}^{N} h_{l, i}^{2}\left\|u_{i}\right\|_{2, \Omega_{i}}^{2}\right)^{1 / 2}\left\|w_{l}\right\|_{l} .
\end{aligned}
$$

Proof. The proof of the first inequality (1) refers to [11]. We only need to prove (2). Let $\gamma_{m(i)}$ be a mortar, $\delta_{m(j)}=\gamma_{m(i)}$ is a nonmortar, then

$$
\int_{\delta_{m(j)}} \frac{\partial u}{\partial n}\left[\bar{w}_{l}\right] d s=\int_{\delta_{m(j)}}\left(\frac{\partial u}{\partial n}-\varphi\right)\left[\bar{w}_{l}\right] d s, \quad \forall \varphi \in S_{l}\left(\delta_{m(j)}\right) .
$$

By a result in [6], we know that there exists a $\varphi \in S_{l}\left(\delta_{m(j)}\right)$ such that

$$
\left\|\frac{\partial u}{\partial n}-\varphi\right\|_{H^{-1 / 2}\left(\delta_{m(j)}\right)} \leqslant C h_{l, j}\left\|\frac{\partial u}{\partial n}\right\|_{H^{1 / 2}\left(\delta_{m(j)}\right)} .
$$

So, by (19),(20) and the trace theorem, we get

$$
\begin{aligned}
& \left|\int_{\delta_{m(j)}} \frac{\partial u}{\partial n}\left[\bar{w}_{l}\right] d s\right| \\
& \leqslant \inf _{\varphi \in S_{l}\left(\delta_{m(j)}\right)}\left\|\frac{\partial u}{\partial n}-\varphi\right\|_{H^{-1 / 2}\left(\delta_{m(j)}\right)}\left\|\bar{w}_{l, j}-\bar{w}_{l, i}\right\|_{H^{1 / 2}\left(\delta_{m(j)}\right)} \\
& \leqslant C h_{l, j}\left\|u_{j}\right\|_{2, \Omega_{j}}\left(\left\|\bar{w}_{l, j}\right\|_{H^{1 / 2}\left(\delta_{m(j)}\right)}+\left\|\bar{w}_{l, i}\right\|_{H^{1 / 2}\left(\gamma_{m(i)}\right)}\right) \\
& \leqslant C h_{l, j}\left\|u_{j}\right\|_{2, \Omega_{j}}\left(\left\|w_{l, i}\right\|_{l, i}+\left\|w_{l, j}\right\|_{l, j}\right) .
\end{aligned}
$$

Summing over all nonmortar $\delta_{m(j)}$ yields $(2)$.

Finally, combining Lemma 3.2-3.4, we get

Theorem 3.1. Let $u$ and $u_{l}$ be the solution of (2) and (14), respectively, then

$$
\left\|u-u_{l}\right\|_{l}^{2} \leqslant C \sum_{i=1}^{N} h_{l, i}^{2}\|u\|_{2, \Omega_{i}}^{2} .
$$




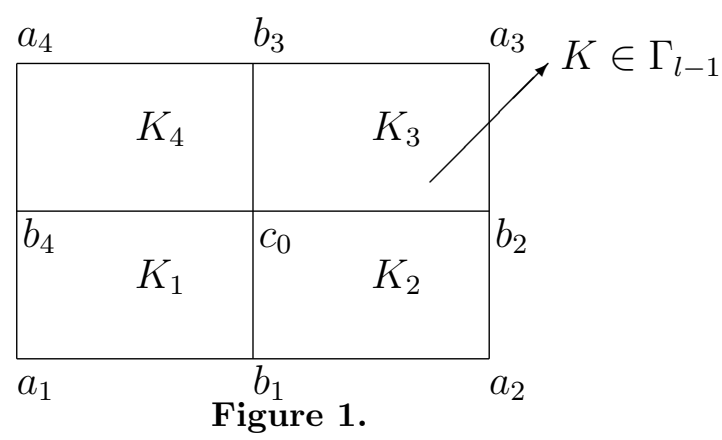

\section{Multigrid algorithm}

Due to the nonnestedness of mesh spaces, we first introduce an intergrid transfer operator. Then a multigrid iterative method is suggested for solving (14). Some preliminary lemmas are given, which will be used to derive the convergence results of the multigrid algorithm. In the following, we always assume that the mesh sizes $h_{l, i}$ for all $i$ are comparable. The reason is that the convergence of multigrid always requires similar mesh parameters.

We define the intergrid transfer operator $J_{l}^{i}: V_{l-1, i}\left(\Omega_{i}\right) \rightarrow V_{l, i}\left(\Omega_{i}\right)$.

Let $K$ be a rectangle in $\Gamma_{l}^{i}$ (cf. Fig. 1$), a_{i}(i=1, \ldots, 4)$ are its vertices, $c_{0}$ is the center, $b_{i}(i=1, \ldots, 4)$ are the midpoints of four edges. Connecting $b_{1}, b_{3}$ and $b_{2}, b_{4}$, we get four equal rectangles $K_{i}(i=1, \ldots, 4)$ in $\Gamma_{l}^{i}$ with $c_{i}(i=1, \ldots, 4)$ being its center. The lengths of $a_{1} a_{2}, a_{1} a_{4}$ are denoted by $2 h_{1}, 2 h_{2},|K|$ is the area of $K$.

For every $v \in V_{l-1, i}$, define $J_{l}^{i} v$ on $K_{1}$ as follows:

$$
\begin{aligned}
\left(J_{l}^{i} v\right)\left(c_{0}\right) & =v\left(c_{0}\right), \\
\left(J_{l}^{i} v\right)\left(a_{1}\right) & =v\left(a_{1}\right), \\
\left(J_{l}^{i} v\right)\left(b_{1}\right) & =\frac{1}{2}\left(v\left(a_{1}\right)+v\left(a_{2}\right)\right), \\
\left(J_{l}^{i} v\right)\left(b_{4}\right) & =\frac{1}{2}\left(v\left(a_{1}\right)+v\left(a_{4}\right)\right), \\
\frac{1}{\left|K_{1}\right|} \int_{K_{1}} \frac{\partial^{2} J_{l}^{i} v}{\partial x^{2}} d x d y & =\frac{1}{|K|} \int_{K} \frac{\partial^{2} v}{\partial x^{2}} d x d y, \\
\frac{1}{\left|K_{1}\right|} \int_{K_{1}} \frac{\partial^{2} J_{l}^{i} v}{\partial y^{2}} d x d y & =\frac{1}{|K|} \int_{K} \frac{\partial^{2} v}{\partial y^{2}} d x d y .
\end{aligned}
$$

Accordingly, we can define the operator $J_{l}: \tilde{V}_{l-1} \rightarrow \tilde{V}_{l}$ as

$$
J_{l} v=\left(J_{l}^{1} v_{1}, \ldots, J_{l}^{N} v_{N}\right) \in \tilde{V}_{l}, \quad \forall v=\left(v_{1}, \ldots, v_{N}\right) \in \tilde{V}_{l-1} .
$$

Lemma 4.1. For $v \in V_{l-1}$, it holds that

(1) $\left\|J_{l}^{i} v\right\|_{l, i} \leqslant C\|v\|_{l-1, i}$,

(2) $\left\|J_{l}^{i} v-v\right\|_{0} \leqslant C h_{l}\|v\|_{l-1, i}$,

(3) $\left\|J_{l}^{i} v-v\right\|_{0, \gamma_{m}} \leqslant C h_{l, i}^{1 / 2}\|v\|_{l-1, i}$,

where $\gamma_{m}$ is an edge of $\Omega_{i}$.

Proof. We refer (1), (2) to [16]. As for (3), the scaling argument [11] gives

$$
\left\|J_{l}^{i} v-v\right\|_{0, \gamma_{m}}^{2} \leqslant C \frac{1}{h_{l, i}}\left\|J_{l, i} v-v\right\|_{0}^{2}+h_{l, i}\left\|J_{l, i} v-v\right\|_{l}^{2}
$$


Inserting (1), (2) into the above inequality yields (3).

Let $\left\{y_{l}^{i}\right\}$ denote the nodes of $\delta_{m(j)}$ and the operator $X \rightarrow \tilde{W}_{l}$ is defined by

$$
\left(\Xi_{l, \delta_{m(j)}}(v)\right)\left(y_{l}^{i}\right)= \begin{cases}\left(\pi_{l, m}\left(\left.v\right|_{\gamma_{m(i)}}-\left.v\right|_{\delta_{m(j)}}\right)\right)\left(y_{l}^{i}\right), & y_{l}^{i} \in \delta_{m(j)} \\ 0 & \text { otherwise }\end{cases}
$$

It is not difficult to check that for any $v \in \tilde{W}_{l}$, we have

$$
v^{*}=v+\sum_{m=1}^{M} \Xi_{l, \delta_{m(j)}}(v) \in W_{l} .
$$

We now define the intergrid transfer operator $I_{l}: V_{l-1} \rightarrow V_{l}$ which will appear in the following multigrid algorithm. For any $v \in V_{l-1}$, define

$$
I_{l} v=J_{l} v+\sum_{m=1}^{M} \Xi_{l, \delta_{m(j)}}\left(\overline{J_{l} v}\right) .
$$

It is easy to check that

$$
I_{l} v \in V_{l} \text {. }
$$

Lemma 4.2. For the operator $I_{l}$, we have

(1) $\left\|I_{l} v\right\|_{l} \leqslant C\|v\|_{l-1}$,

(2) $\left\|v-I_{l} v\right\|_{0} \leqslant C h_{l}\|v\|_{l-1}, \quad \forall v \in V_{l}$.

Proof. By Lemma 4.1, we get

$$
\begin{aligned}
\left\|I_{l} v\right\|_{l}^{2} & \leqslant C\left(\left\|J_{l} v\right\|_{l}^{2}+\sum_{m=1}^{M}\left\|\Xi_{l, \delta_{m(j)}}\left(\overline{J_{l} v}\right)\right\|_{l}^{2}\right) \\
& \leqslant C\left(\|v\|_{l-1}^{2}+\sum_{m=1}^{M}\left\|\Xi_{l, \delta_{m(j)}}\left(\overline{J_{l} v}\right)\right\|_{l}^{2}\right) .
\end{aligned}
$$

For each nonmortar edge $\delta_{m(j)}$, we can derive

$$
\begin{aligned}
\left\|\Xi_{l, \delta_{m(j)}}\left(\overline{J_{l} v}\right)\right\|_{l}^{2} & \leqslant C \sum_{y_{l}^{i}}\left(\Xi_{l, \delta_{m(j)}}\left(\overline{J_{l} v}\right)\left(y_{l}^{i}\right)\right)^{2} \\
& =C \sum_{y_{l}^{i}}\left(\pi_{l, m}\left(\left.\overline{J_{l} v}\right|_{\gamma_{m(i)}}-\left.\overline{J_{l} v}\right|_{\delta_{m(j)}}\right)\right)^{2}\left(y_{l}^{i}\right) \\
& \leqslant C h_{l}^{-1}\left\|\pi_{l, m}\left(\left.\overline{J_{l} v}\right|_{\gamma_{m(i)}}-\left.\overline{J_{l} v}\right|_{\delta_{m(j)}}\right)\right\|_{0, \delta_{m(j)}}^{2} \\
& \leqslant C h_{l}^{-1}\left\|\left.\overline{J_{l} v}\right|_{\gamma_{m(i)}}-\left.\overline{J_{l} v}\right|_{\left.\delta_{m(j)}\right)}\right\|_{0, \gamma_{m}} \\
& \leqslant C h_{l}^{-1}\left(\left\|\left.\overline{J_{l} v}\right|_{\gamma_{m(i)}}-\left.\bar{v}\right|_{\gamma_{m(i)}}\right\|_{0, \gamma_{m}}^{2}+\left\|\left.\bar{v}\right|_{\gamma_{m(i)}}-\left.\bar{v}\right|_{\delta_{m(j)}}\right\|_{0, \gamma_{m}}^{2}\right. \\
& \left.\quad+\left\|\left.\bar{v}\right|_{\delta_{m(j)}}-\left.\overline{J_{l} v}\right|_{\delta_{m(j)}}\right\|_{0, \gamma_{m}}^{2}\right) \\
& \hat{=} C h_{l}^{-1}\left(I_{1}+I_{2}+I_{3}\right) .
\end{aligned}
$$

By the interpolation theory and Lemma 4.1, we have

$$
I_{1} \leqslant C\left\|J_{l}^{i} v-v\right\|_{0, \gamma_{m}}^{2} \leqslant C h_{l}\|v\|_{l-1, i}^{2} .
$$


Similarly

$$
I_{3} \leqslant C h_{l}\|v\|_{l-1, j}^{2}
$$

Now we estimate $I_{2}$. Let

$$
\left.\eta \hat{=} \bar{v}\right|_{\gamma_{m(i)}}-\left.\bar{v}\right|_{\delta_{m(j)}}
$$

Owing to $v \in V_{l-1}$

$$
(\eta, \eta)=(\eta, \eta-\mu), \quad \forall \mu \in S_{l-1}\left(\delta_{m(j)}\right),
$$

where $(\cdot, \cdot)$ denotes the $L^{2}\left(\delta_{m(j)}\right)$ inner product. By the approximation theory and the trace theorem, we have

$$
\begin{aligned}
I_{2} & \leqslant \inf _{\mu \in S_{l-1}\left(\delta_{m(j)}\right)}\|\eta-\mu\|_{0, \delta_{m(j)}}^{2} \\
& \leqslant C h_{l}\|\eta\|_{1 / 2, \gamma_{m}}^{2} \leqslant C h_{l}\|v\|_{\Omega_{i} \cup \Omega_{j}}^{2} .
\end{aligned}
$$

Summing up all $\gamma_{m}$ yields (1).

We prove (2). In fact, from the definition of $I_{l}$ and Lemma 4.1, it follows that

$$
\begin{aligned}
\left\|v-I_{l} v\right\|_{0}^{2} & \leqslant C\left(\left\|v-J_{l} v\right\|_{0}^{2}+\sum_{m=1}^{M}\left\|\Xi_{l, \delta_{m(j)}}\left(\overline{J_{l} v}\right)\right\|_{0}^{2}\right) \\
& \leqslant C\left(h_{l}\|v\|_{l-1}^{2}+\sum_{m=1}^{M}\left\|\Xi_{l, \delta_{m(j)}}\left(\overline{J_{l} v}\right)\right\|_{0}^{2}\right) .
\end{aligned}
$$

By a similar argument as in the proof of (1), we can derive

$$
\left\|\Xi_{l, \delta_{m(j)}}\left(\overline{J_{l} v}\right)\right\|_{0} \leqslant C h_{l}\|v\|_{\Omega_{i} \cup \Omega_{j}}
$$

which in conjunction with (29) yields (2).

Now we can apply the theory given in [9] to construct our multigrid algorithm. An optimal $W$-cycle multigrid algorithm is established, i.e., the convergence rate is independent of the mesh size $h_{l}$ and mesh level $L$. Meanwhile, a variable $V$-cycle multigrid preconditioner is constructed, which results in a preconditioned system with a uniformly bounded condition number.

We define some operator: $A_{l}$ denotes the operator on $V_{l}$, which is defined by

$$
\left(A_{l} u, v\right)=a_{l}(u, v) \quad \forall u, v \in V_{l} .
$$

Moreover, define the operators $P_{l-1}: V_{l} \rightarrow V_{l-1}$ and $P_{l-1}^{0}: V_{l} \rightarrow V_{l-1}$ by

$$
a_{l-1}\left(P_{l-1} u, v\right)=a_{l}\left(u, I_{l} v\right) \quad \forall u \in V_{l}, \quad v \in V_{l-1}, \quad l=2, \ldots, L,
$$

and

$$
\left(P_{l-1}^{0} u, v\right)_{l-1}=\left(u, I_{l} v\right) \quad \forall u \in V_{l}, \quad v \in V_{l-1}, \quad l=2, \ldots, L .
$$

A general multigrid operator $B_{l}: V_{l} \rightarrow V_{l}$ can be defined recursively as follows.

\section{Multigrid Algorithm}

Let $1 \leqslant l \leqslant L$ and $p$ be a positive interger. Set $B_{1}=A_{1}^{-1}$. Assume that $B_{l-1}$ has been defined and define $B_{l} g$ for $g \in V_{l}$ by

(1) Set $x_{0}=0$ and $q^{0}=0$. 
(2) Define $x^{k}$ for $k=1, \ldots, m(l)$ by

$$
x^{k}=x^{k-1}+R_{l}^{(l+m(l))}\left(g-A_{l} x^{k-1}\right) .
$$

(3) Define $y^{m(l)}=x^{m(l)}+I_{l} q^{p}$, where $q^{i}$ for $i=1, \ldots, p$ is determined by

$$
q^{i}=q^{i-1}+B_{l-1}\left(P_{l-1}^{0}\left(g-A_{l} x^{m(l)}\right)-A_{l-1} q^{i-1}\right) .
$$

(4) Define $y^{l}$ for $k=m(l)+1, \ldots, 2 m(l)$ by

$$
y^{k}=y^{k-1}+R_{l}^{(k+m(l))}\left(g-A_{l} y^{k-1}\right) .
$$

(5) Set $B_{l} g=y^{2 m(l)}$,

where $R_{l}=\lambda_{l}^{-1}, \lambda_{l}$ is the largest eigenvalue of $A_{l}$.

Remark 4.1. Here we only use the Richardson iteration as a smoothing procedure, but by the same argument as in [13] we can use other iteration methods like the Jacobi iteration to our multigrid method.

Remark 4.2. In the above algorithm, $m(l)$ gives the number of pre- and post-smoothing steps and can vary as a function of $l$. If $p=1$, we have a $V$-cycle method. If $p=2$, we have a $W$-cycle method. Other versions of multigrid methods without pre- or post-smoothing iterative can be analyzed similarly. A variable $V$-cycle multigrid method is one in which the number of smoothing $m(l)$ increases exponentially as $l$ decreases.

According to [9], an important ingredient in the multigrid analysis is the following regularity and approximation assumption.

(A1). There exists an $\alpha \in(0,1]$ such that

$$
\left|a_{l}\left(v-I_{l} P_{l-1} v, v\right)\right| \leqslant C\left(\frac{\left\|A_{l} v\right\|_{0}^{2}}{\lambda_{l}}\right)^{\alpha} a_{l}(v, v)^{1-\alpha} \quad \forall v \in V_{l},
$$

where $\lambda_{l}$ is the maximum eigenvalue of $A_{l}$.

\section{Convergence analysis}

The convergence rate for the above multigrid algorithm on the $l$ th level is measured by a convergence factor $\delta_{l}$ satisfying

$$
\left|a_{l}\left(\left(I-B_{l} A_{l}\right) v, v\right)\right| \leqslant \delta_{l} a_{l}(v, v) .
$$

Following [9], we state two propositions:

Proposition 5.1 ( $W$-cycle). Under the conditions (A1), if $p=2$ and $m(l)=m$ is large enough, then the convergence factor in (5.1) is

$$
\delta_{l}=\frac{C}{C+m^{\alpha}}
$$


Proposition 5.2 (variable $V$-cycle preconditioner). Assume that (A1) is valid and the number of smoothings $m(l)$ increases as $l$ decreases in such a way that

$$
\beta_{0} m(l) \leqslant m(l-1) \leqslant \beta_{1} m(l)
$$

holds with $1<\beta_{0} \leqslant \beta_{1}$. Then there exists an $M>0$ independent of $L$ such that

$$
C_{0}{ }^{-1} a_{L}(v, v) \leqslant a_{L}\left(B_{L} A_{L} v, v\right) \leqslant C_{0} a_{L}(v, v) \quad \forall v \in V_{L}
$$

with $C_{0}=\frac{M+m(L)^{1 / 2}}{m(L)^{1 / 2}}$.

In the following, we will prove that (A1) holds for the Wilson mortar element method.

Define the operator $\bar{P}_{l-1}: V_{l} \rightarrow W_{l-1}$ as follows:

$$
a_{l-1}\left(\bar{P}_{l-1} u, v\right)=a_{l}\left(u, I_{l} v\right) \quad \forall u \in V_{l}, \quad v \in W_{l-1}, \quad l=2, \ldots, L .
$$

Then we have

Lemma 5.1. For the operator $\bar{P}_{l-1}$, it holds that

$$
\left\|v-I_{l} \bar{P}_{l-1} v\right\|_{l} \leqslant C h_{l}\left\|A_{l} v\right\|_{0} \quad \forall v \in V_{l} .
$$

Proof. Consider the following auxiliary problem

$$
\begin{cases}-\triangle \xi=g & \text { in } \Omega, \\ \xi=0 & \text { on } \partial \Omega\end{cases}
$$

where $g \hat{=} A_{l} v$.

Obviously, $v$ is the Wilson mortar finite element approximation of $\xi$ in the space $V_{l}$. So by Lemma 3.2-3.4 and the regularity assumption (H1) we get

$$
\|\xi-v\|_{l} \leqslant C h_{l}\|\xi\|_{2} \leqslant C h_{l}\left\|A_{l} v\right\|_{0} .
$$

Let $v_{l-1} \in W_{l-1}$ be the solution of the following variational problem

$$
a_{l-1}\left(v_{l-1}, \phi\right)=(g, \phi) \quad \forall \phi \in W_{l-1} .
$$

By a result in [6], we have

$$
\left\|\xi-v_{l-1}\right\|_{l-1} \leqslant C h_{l}\|\xi\|_{2} \leqslant C h_{l}\left\|A_{l} v\right\|_{0}
$$

An application of the triangle inequality yields

$$
\begin{aligned}
\left\|v-I_{l} \bar{P}_{l-1} v\right\|_{l} \leqslant & \|v-\xi\|_{l}+\left\|\xi-I_{l} \bar{P}_{l-1}\right\|_{l} \\
\leqslant & \|v-\xi\|_{l}+\left\|\xi-v_{l-1}\right\|_{l-1}+\left\|\left(I-I_{l}\right) v_{l-1}\right\|_{l} \\
& \quad+\left\|I_{l}\left(v_{l-1}-\bar{P}_{l-1} v\right)\right\|_{l} \\
& \hat{=} \sum_{i=1}^{4} I_{i} .
\end{aligned}
$$

By (38) and (39), we have

$$
I_{i} \leqslant C h_{l}\left\|A_{l} v\right\|_{0} \quad i=1,2
$$


For $I_{3}$, by the definition of $I_{l}$ and the fact that

$$
J_{l} v_{l-1}=v_{l-1} \quad \forall v_{l-1} \in W_{l-1},
$$

it follows that

$$
\left(I-I_{l}\right) v_{l-1}=\sum_{m=1}^{M} \Xi_{l, \delta_{m(j)}}\left(J_{l} v_{l-1}\right)=\sum_{m=1}^{M} \Xi_{l, \delta_{m(j)}}\left(v_{l-1}\right) .
$$

Using a similar argument in Lemma 4.2, we can derive

$$
\left\|\left(I-I_{l}\right) v_{l-1}\right\|_{l}^{2} \leqslant C \sum_{i=1}^{M}\left\|\left.v_{l-1}\right|_{\gamma_{m(i)}}-\left.v_{l-1}\right|_{\delta_{m(j)}}\right\|_{1 / 2, \gamma_{m}}^{2} .
$$

Noting that $\xi \in H_{0}^{1}(\Omega)$, we have

$$
\begin{aligned}
\left\|\left(I-I_{l}\right) v_{l-1}\right\|_{l}^{2} & \leqslant C \sum_{m=1}^{M}\left\|\left.\left(v_{l-1}-\xi\right)\right|_{\gamma_{m(i)}}-\left.\left(v_{l-1}-\xi\right)\right|_{\delta_{m(j)}}\right\|_{1 / 2, \gamma_{m}}^{2} \\
& \leqslant \sum_{m=1}^{M} C\left\|v_{l-1}-\xi\right\|_{1 / 2, \partial \Omega_{i}}^{2}+C\left\|v_{l-1}-\xi\right\|_{1 / 2, \partial \Omega_{j}}^{2} \\
& \leqslant C\left\|v_{l-1}-\xi\right\|_{1, \Omega}^{2}+C\left\|v_{l-1}-\xi\right\|_{1, \Omega}^{2} \\
& \leqslant C h_{l}^{2}\|\xi\|_{2}^{2} \leqslant C h_{l}^{2}\left\|A_{l} v\right\|_{0}^{2}
\end{aligned}
$$

which gives the estimation of $I_{3}$.

For the last term $I_{4}$, using Lemma 4.2, we can estimate it as follows:

$$
\begin{aligned}
I_{4} & \leqslant C\left\|v_{l-1}-\bar{P}_{l-1} v\right\|_{l-1} \\
& =C \sup _{\varphi \in W_{l-1}} \frac{a_{l-1}\left(v_{l-1}-\bar{P}_{l-1} v, \varphi\right)}{\|\varphi\|_{l-1}} \\
& =C \sup _{\varphi \in W_{l-1}} \frac{\left(g, \varphi-I_{l} \varphi\right)}{\|\varphi\|_{l-1}} \\
& \leqslant C h_{l}\|g\|_{0}=C h_{l}\left\|A_{l} v\right\|_{0} .
\end{aligned}
$$

Combining (41),(45),(46), we complete the proof.

Lemma 5.2. For the operator $P_{l-1}, \bar{P}_{l-1}$, we have

$$
\left\|\left(\bar{P}_{l-1}-P_{l-1}\right) v\right\|_{l-1} \leqslant C h_{l}\left\|A_{l} v\right\|_{0} \quad \forall v \in V_{l} .
$$

Proof. Consider the auxiliary problem (37) again. Let $w_{l-1}$ be the solution of the following variational problem

$$
a_{l-1}\left(w_{l-1}, \varphi\right)=(g, \varphi), \quad \varphi \in V_{l-1} .
$$

Hereafter we use the same notations as in the previous proof. We can write

$$
\begin{aligned}
\left(\bar{P}_{l-1}-P_{l-1}\right) v= & v_{l-1}+\left(\bar{P}_{l-1} v-v_{l-1}\right)-w_{l-1} \\
& -\left(P_{l-1} v-w_{l-1}\right) .
\end{aligned}
$$


An application of the triangle inequality, (39), (46) and Lemma 3.2-3.4 yields

$$
\begin{aligned}
\left\|\left(\bar{P}_{l-1}-P_{l-1}\right) v\right\|_{l-1} \leqslant & \left\|v_{l-1}-w_{l-1}\right\|_{l-1}+\left\|\bar{P}_{l-1} v-v_{l-1}\right\|_{l-1} \\
& +\left\|P_{l-1} v-w_{l-1}\right\|_{l-1} \\
\leqslant & \left\|v_{l-1}-\xi\right\|_{l-1}+\left\|w_{l-1}-\xi\right\|_{l-1} \\
& +\left\|\bar{P}_{l-1} v-v_{l-1}\right\|_{l-1}+\left\|P_{l-1} v-w_{l-1}\right\|_{l-1} \\
\leqslant & C h_{l}\left\|A_{l} v\right\|_{0}+\left\|P_{l-1} v-w_{l-1}\right\|_{l-1} .
\end{aligned}
$$

So we only need to estimate the last term in above the inequality, that is

$$
\begin{aligned}
\left\|P_{l-1} v-w_{l-1}\right\|_{l-1} & =\sup _{\varphi \in V_{l-1}} \frac{a_{l-1}\left(w_{l-1}-P_{l-1} v, \varphi\right)}{\|\varphi\|_{l-1}} \\
& =\sup _{\varphi \in V_{l-1}} \frac{\left(g, \varphi-I_{l} \varphi\right)}{\|\varphi\|_{l-1}} \\
& \leqslant C h_{l}\|g\|_{0}=C h_{l}\left\|A_{l} v\right\|_{0} .
\end{aligned}
$$

Combining (48), (49) yields Lemma 5.2.

Now we are in a position to prove the assumption (A1). In fact, by Lemmas 5.1, 5.2 and 4.2 , we can derive

$$
\begin{aligned}
\left|a_{l}\left(\left(I-I_{l} P_{l-1}\right) v, v\right)\right| & \leqslant \|\left(I-I_{l} P_{l-1} v\left\|_{l}\right\| v \|_{l}\right. \\
& \leqslant\left(\left\|\left(I-I_{l} \bar{P}_{l-1}\right) v\right\|_{l}+\left\|I_{l}\left(\bar{P}_{l-1}-P_{l-1}\right) v\right\|_{l}\right)\|v\|_{l} \\
& \leqslant C h_{l}\left\|A_{l} v\right\|_{0}\|v\|_{l} \\
& =C\left(\frac{\left\|A_{l} v\right\|_{0}^{2}}{\lambda_{l}}\right)^{1 / 2} a_{l}(v, v)^{1 / 2}
\end{aligned}
$$

which means that (A1) is vaild with $\alpha=1 / 2$.

Remark 5.1. We can easily extend the results of this paper to other nonconforming elements like the Carey triangular element (see $[10,15])$.

Remark 5.2. We can also choose the mortar bilinear element space as the coarse mesh correction space. In this case, using a similar technique developed in Section 5, we can show that the main results in this paper are still valid. This alternative approach might have some merits in implementations.

\section{References}

[1] Y. Achdou and Y. A. Kuznetsov, Subtructuring preconditioners for finite element methods on nonmatching grids, East-West J. Numer. Math., 3 (1995), pp. 1-28.

[2] Y. Achdou, Y. A. Kuznetsov, and O. Pironneau, Substructuring preconditioner for the $q_{1}$ mortar element method, Numer. Math., 71 (1995), pp. 419-449.

[3] Y. Achdou, Y. Maday, and O. B. Widlund, Iterative substructing preconditionres for mortar element methods in two dimensions, SIAM J. Numer. Anal., 36 (1999), pp. 551-580.

[4] F. Ben Belgacem, The mortar finite element method with lagrange multipliers, Numer. Math., 84 (1999), pp. 173-197. 
[5] F. Ben Belgacem and Y. Maday, The mortar element method for three dimensional finite elements, RAIAO, 31 (1997), pp. 289-302.

[6] F. Ben Belgacem, Y. Maday, and A. T. Patera, A new nonconforming approach to domain decomposition: the mortar element method, in: Nonlinear Partial Differential Equations and Their Applications, College de France Seminar (H. Brezis and J. L. Lions, eds.), vol. XI, No. 299 of Pitman Research Notes in Mathematics, Longman Scientific Technical, 1994, .

[7] D. Braess and W. Dahmen, Stability estimates of the mortar finite element method for 3-dimensional problems, East-West J. Numer. Math., 6 (1998), pp. 249-263.

[8] D. Braess, W. Dahmen, and C. Wieners, A multigrid algorithm for the mortar finite element method, to appear in SIAM J. Numer. Anal., 2000.

[9] J. H. Bramble, J. E. Pascial, and J. Xu, The analysis of multigrid algorithms with nonnested spaces and noninherited quadratic forms, Math. Comp., 56 (1991), pp. 1-34.

[10] G. Carey, An analysis of finite element equation and mesh subdivision, Comput. Methods. Appl. Mech. Engrg., 9 (1976), pp. 165-179.

[11] P. G. Ciarlet, The Finite Element Method for Elliptic Problems, North-Holland, Amsterdam, 1978.

[12] M. Dryja, An iterative substructuring method for elliptic mortar finite element problems with discontinous coefficients, in: Domain Decomposition Methods 10 (J. Mandel, C. Farhat, and X. C. Cai, eds.), vol. 218 of Contemp. Math., 1998, pp. 94-103.

[13] J. Gopalakrishnan and J. P. Pasciak, Multigrid for the mortar finite element method, to appear in SIAM J. Numer. Anal., 2000.

[14] L. Marcinkowski, The mortar element method with locally nonconforming elements, BIT, 39 (1999), pp. 716-739.

[15] Z.-C. Shi, Convergence properties of two nonconforming finite elements, Comput. Methods Appl. Mech. Engrg., 48 (1985), pp. 123-139.

[16] Z.-C. Shi and B. Jiang, Multigrid method for wilson nonconforming finite element with numerical integration, in: Domain Decomposition Methods and Related Topics, Symposium of Research Institute for Mathematical Sciences, Kyoto University, 1996, pp. 103-121.

[17] B. Wohlmuth, A multigrid method for saddle point problems arising from mortar finite element discretizations, ETNA, 11 (2000), pp. 43-54.

[18] O. C. Zienkiewicz and R. L. Taylor, The Finite Element Methods, 4th edn., Mcgraw-Hill, London, 1989. 